ARTICLE

\title{
Nickel-catalyzed reductive coupling of homoenolates and their higher homologues with unactivated alkyl bromides
}

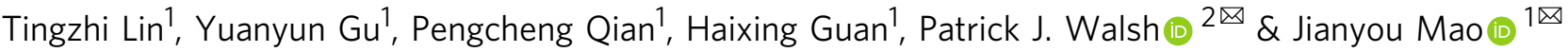

\begin{abstract}
The catalytic generation of homoenolates and their higher homologues has been a longstanding challenge. Like the generation of transition metal enolates, which have been used to great affect in synthesis and medicinal chemistries, homoenolates and their higher homologues have much potential, albeit largely unrealized. Herein, a nickel-catalyzed generation of homoenolates, and their higher homologues, via decarbonylation of readily available cyclic anhydrides has been developed. The utility of nickel-bound homoenolates and their higher homologues is demonstrated by cross-coupling with unactivated alkyl bromides, generating a diverse array of aliphatic acids. A broad range of functional groups is tolerated. Preliminary mechanistic studies demonstrate that: (1) oxidative addition of anhydrides by the catalyst is faster than oxidative addition of alkyl bromides; (2) nickel bound metallocycles are involved in this transformation and (3) the catalyst undergoes a single electron transfer (SET) process with the alkyl bromide.
\end{abstract}

\footnotetext{
${ }^{1}$ Technical Institute of Fluorochemistry (TIF), Institute of Advanced Synthesis, School of Chemistry and Molecular Engineering, Nanjing Tech University, 30 South Puzhu Road, Nanjing 211816, China. ${ }^{2}$ Roy and Diana Vagelos Laboratories, Penn/Merck Laboratory for High-Throughput Experimentation, Department of Chemistry, University of Pennsylvania, 231 South 34th Street, Philadelphia, Pennsylvania 19104, USA. ${ }^{凶}$ email: pwalsh@sas.upenn.edu; ias_jymao@njtech.edu.cn
} 
omoenolates and their higher homologs represent important synthetic intermediates that have found broad applications in natural product synthesis and pharmaceutical sciences ${ }^{1-3}$. In comparison to ketones, which exhibit nucleophilic character upon deprotonation of the relatively acidic $\alpha-\mathrm{C}-\mathrm{H}$ 's, the $\beta$ - and $\gamma$-C-Hs are usually unreactive or, in the case of $\alpha, \beta$-unsaturated derivatives, the $\beta$-carbon is electrophilic. Thus, with respect to $\alpha, \beta$-unsaturated carbonyl compounds, the $\beta$-position of homoenolates exhibits umpolung reactivity ${ }^{4-6}$. Despite great progress in the generation and catalytic reactions of homoenolates 7,8 , the demand for more straightforward approaches to these reactive species and their higher homologs remains high.

Early routes for stoichiometric generation of homoenolates include (a) $\beta$-deprotonation of camphenilone with $\mathrm{KO} t \mathrm{Bu}$ by Nickon and Lambert ${ }^{9}$; (b) $\mathrm{TiCl}_{4}$-mediated ring opening of silyloxycyclopropanes by Nakamura and Kuwajima ${ }^{10}$; (c) Brønsted base-mediated metalation of allyl carbamates ${ }^{11}$; and (d) directed $\beta$-metalation of $N, N$-diisopropyl amides by Beak and colleagues $^{12,13}$, among others ${ }^{14-20}$. The generation and reactions of $\gamma$-metallocarbonyls, however, remains relatively unexplored ${ }^{21}$.

Kuwajima, Nakamura, and their colleagues ${ }^{22}$ pioneered catalytic reactions of palladium-bound homoenolates derived from the ring opening of cyclopropanols. The Pd-homoenolates coupled with aryl halides to furnish $\beta$-arylcarbonyl derivatives (Fig. 1a) ${ }^{22}$. However, this ring opening strategy cannot afford $\gamma$-metallocarbonyl analogs. In addition to arylations ${ }^{23,24}$, other transformations such as acylation ${ }^{25}$, benzylation ${ }^{26}$, and allenylation $^{27}$ of palladium-homoenolates were also reported via ring opening of cyclopropanols. In the case of $\beta$-alkylation of homoenolates, only activated alkyl halides have been successfully employed $^{28-31}$. For example, this year the Fu group developed a remarkable Ni-catalyzed coupling of racemic $\beta$-zincated amides with racemic propargylic bromides in a doubly stereoconvergent process to provide amides with high ee and dr (Fig. 1b) ${ }^{32}$. In addition to ring opening of cyclopropanols, directing groupassisted palladium-catalyzed $\beta-\mathrm{C}-\mathrm{H}$ activations are an alternative strategy to generate and functionalize palladium-homoenolates of carboxylic acid derivatives ${ }^{33-36}$. More recently, a palladiumcatalyzed functionalization of $\gamma$-C-H's of carboxylic acid derivatives was realized. This method employed 3,3-disubstituted butyric acid derivatives to circumvent the $\beta-\mathrm{C}-\mathrm{H}$ functionalization pathway (Fig. 1c) ${ }^{21,37}$. To the best of our knowledge, the use of inexpensive nickel catalysts for the generation and functionalization of $\beta$-, or $\gamma$-bound carboxylic acid derivatives remains underdeveloped, especially related to coupling of these intermediates with alkyl groups ${ }^{38,39}$. To circumvent the use of unactivated alkyl halides, Rousseaux and colleagues ${ }^{40}$ developed an elegant alkylation of homoenolates derived from cyclopropanols that involves use of redox active esters (Fig. 1d). In this work, redox active esters undergo in situ decarboxylation to provide alkyl radicals, leading to alkylated homoenolates ${ }^{40}$. To the best of our knowledge, catalytic alkylations of any type of homoenolate, or their higher homologs, with unactivated alkyl halides remains a challenge. This is likely due to the relatively slow oxidative addition of alkyl electrophiles and facile $\beta$-hydride elimination of the resulting intermediates ${ }^{41-44}$.

We have been interested in developing new transformations of homoenolates 45,46 and cross-electrophile coupling of cyclic anhydrides with aryl triflates to afford aryl ketoacids ${ }^{47}$. We were inspired by Yamamoto's stoichiometric reactions of nickel $(0)$ complexes with anhydrides and Rovis's stoichiometric nickel promoted transformation with cyclic anhydrides and $\mathrm{ZnPh}_{2}$ (Fig. 2a) ${ }^{48,49}$. These latter reactions undergo insertion of nickel into the anhydride $\mathrm{C}-\mathrm{O}$ bond followed by decarbonylation to generate a nickel homoenolate. Transmetallation from $\mathrm{ZnPh}_{2}$ is followed by reductive elimination to form the $\mathrm{C}-\mathrm{C}$ bond. Herein, we report a nickel-catalyzed alkylation of homoenolates and their higher homologs via decarbonylation of monocyclic anhydrides (Fig. 2b). The in situ-generated nickel homoenolate species are successfully coupled with unactivated alkyl bromides, producing a diverse array of valuable functionalized acids. In addition to alkylation of nickel bound homoenolates, $\gamma$-alkylation of butyric acid derivatives can also be performed under the same conditions. Compared with Martín's elegant carboxylation of alkyl bromides with $\mathrm{CO}_{2}$, our strategy provides an alternative synthesis of functionalized acids ${ }^{50,51}$. It is noteworthy that functionalized aliphatic acids are popular structures in soaps, dyes, plastics, and many chemicals ${ }^{52}$. They are also useful precursors in coupling reactions ${ }^{53-55}$.

a
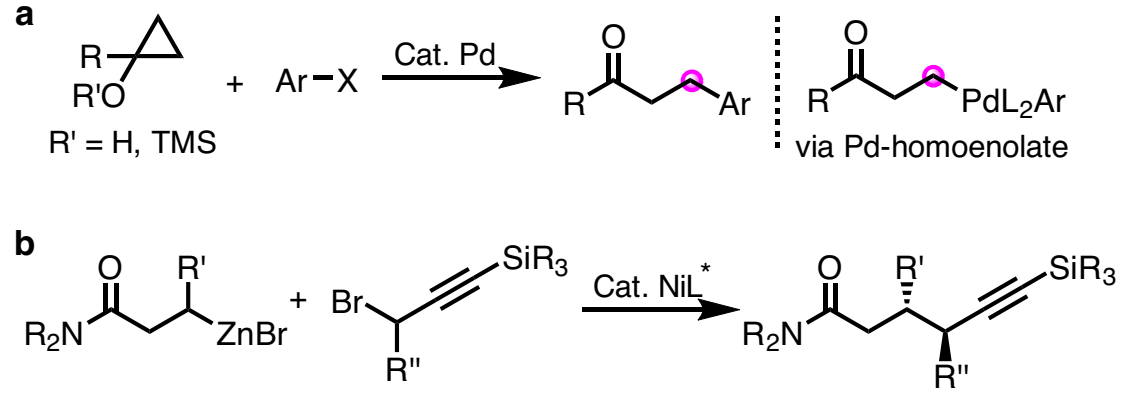

C

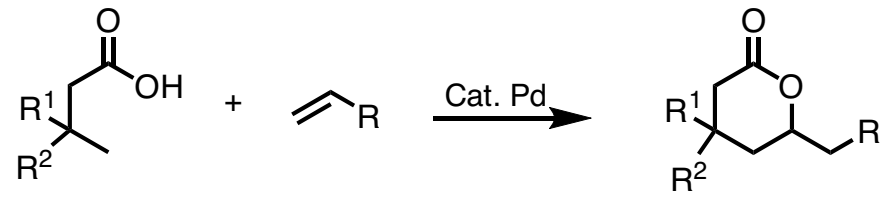

d

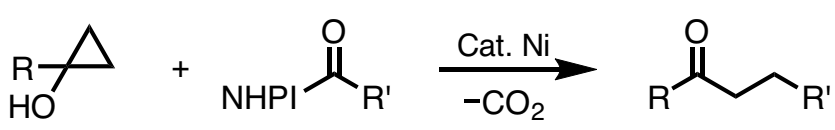

Fig. 1 Metal catalyzed functionalization of homoenolates and their higher homologs. a Palladium-catalyzed arylations of cyclopropanol derivatives. b Ni-catalyzed doubly stereoconvergent coupling. c Palladium-catalyzed functionalization of $\gamma$-C-H's of carboxylic acid derivatives. d Ni-catalyzed alkylations with $\mathrm{N}$-hydroxyphthalimides (NHPI). 


\section{Results}

Reaction development and optimization. To initiate the development of the nickel catalyzed alkylation of homoenolates, we chose succinic anhydride $\mathbf{1 a}$ and 1-bromooctane 2a as model substrates. $\mathrm{Ni}(\mathrm{COD})_{2}$ and bipy were used as catalyst precursors in dimethylacetamide (DMA) at $80^{\circ} \mathrm{C}$ for $12 \mathrm{~h}$. As this is a crosselectrophile coupling reaction, a stoichiometric reducing agent is needed and zinc powder was chosen. After $12 \mathrm{~h}$ under these conditions, the desired decarbonylative product 3aa was obtained in $63 \%$ assay yield (AY) (Table 1, entry 1 , AY, determined by GC analysis of the unpurified reaction mixture with dodecane as internal standard). It should be noted that the nondecarbonylated $\gamma$-keto acid 3aa' was not observed under these conditions. A survey of solvents found DMA was a better choice than $\mathrm{N}, \mathrm{N}$-dimethylformamide (DMF), tetrahydrofuran (THF), THF, or acetonitrile (AY 23-42\%, entries 2-4, see Supplementary Information for details). To increase the AY of 3aa, we next<smiles>O=C1[R]CC(=O)O1</smiles>

i) $\mathrm{Ni}(\mathrm{COD})_{2}(1-1.5$ equiv) neocuproine (1 equiv) dppb ( 0.5 equiv)

ii) 4-fluorostyrene (1 equiv) $\mathrm{Ph}_{2} \mathrm{Zn}$ (2 equiv in THF)

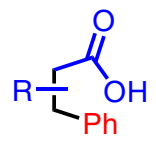<smiles>O=C1CCC(=O)O1</smiles>

$n=1,2$

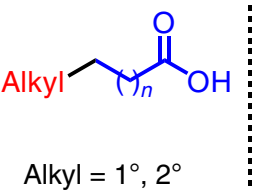

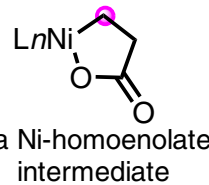

Fig. 2 Nickel-mediated functionalization of cyclic anhydrides. a Rovis's stoichiometric nickel complex with $\mathrm{Ph}_{2} \mathrm{Zn}$. b This work: Ni-catalyzed functionalization with alkyl bromides. explored different nickel sources. Unfortunately, these nickel species afforded lower AY of 3aa (18-52\%, entries 5-8).

To improve the decarbonylative cross-coupling, we turned to examination of nitrogen-based ligands. Under the conditions of entry 1, the parent bipy outperformed all others examined (the complete optimization data is presented in the Supplementary Information). We next examined the effect of concentration on the AY. Increasing the concentration from 0.4 to $2.0 \mathrm{M}$ had a beneficial impact, affording the desired product in $87 \%$ AY at 1.3 $\mathrm{M}$ (entries 1 and 9-12). When 1-chloro- or 1-iodohexane were used in place of 1-bromohexane, the AY were lower than $40 \%$ (see Supplementary Information). Therefore, our optimized reaction conditions are 1.5 equiv of anhydride 1a, 1 equiv of alkyl bromide $2 \mathrm{a}, 2$ equiv of zinc powder, $10 \mathrm{~mol} \% \mathrm{Ni}(\mathrm{COD})_{2}$, and $15 \mathrm{~mol} \%$ bipy in DMA at $80^{\circ} \mathrm{C}$ for $12 \mathrm{~h}$.

Substrate scope. With the optimized reaction conditions in hand (Table 1, entry 11), we next explored the scope of the alkyl bromides. In general, a wide range of primary and secondary alkyl bromides participated in this transformation to afford the acid products in good to excellent yields with very good functional group tolerance (Table 2). We first chose the parent succinic anhydride 1a the homoenolate precursor to couple with a variety of primary alkyl bromides. Simple long-chain 1-bromooctane 2a underwent this coupling to afford 3aa in $84 \%$ isolated yield. More challenging functionalized alkyl bromides bearing benzodihydrofuran, ester, imide, and ether functional groups were compatible under our conditions, generating the corresponding products 3ab-3af in $68-91 \%$ yield.

We were also interested in using glutaric anhydride $\mathbf{1 b}$ Fortunately, glutaric anhydride underwent the decarbonylative alkylation smoothly, providing access to $\gamma$-alkylated acids under mild conditions. Glutaric anhydride reacted with a diverse array of primary alkyl bromides, including functionalized substrates, exhibiting good reactivity and furnishing the desired acids in

Table 1 Optimization of the reaction conditions ${ }^{a}$.

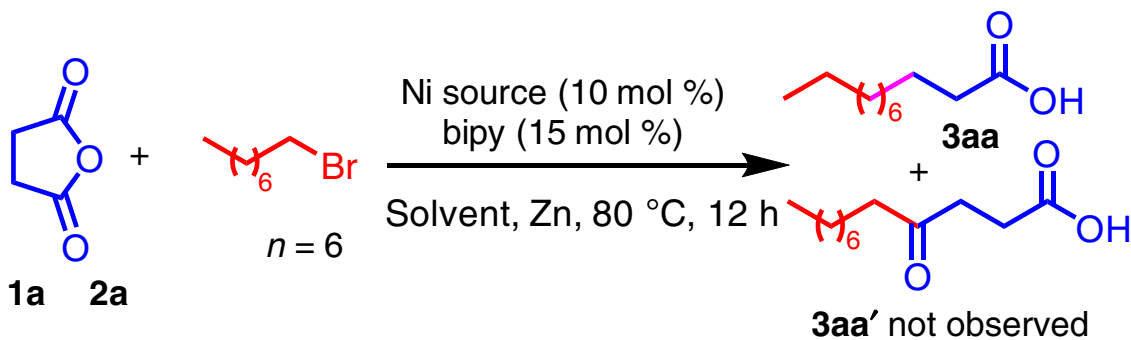

\begin{tabular}{|c|c|c|c|c|}
\hline Entry & Solvent & Catalyst & Conc./M & $A Y^{b}$ \\
\hline 1 & DMA & $\mathrm{Ni}(C O D)_{2}$ & 0.4 & 63 \\
\hline 2 & DMF & $\mathrm{Ni}(\mathrm{COD})_{2}$ & 0.4 & 42 \\
\hline 3 & THF & $\mathrm{Ni}(C O D)_{2}$ & 0.4 & 34 \\
\hline 5 & DMA & $\mathrm{Nil}_{2}$ & 0.4 & 52 \\
\hline 6 & DMA & $\mathrm{DME} \bullet \mathrm{NiBr}_{2}$ & 0.4 & 23 \\
\hline 7 & DMA & $\mathrm{NiCl}_{2} \cdot 6 \mathrm{H}_{2} \mathrm{O}$ & 0.4 & 27 \\
\hline 10 & DMA & $\mathrm{Ni}(\mathrm{COD})_{2}$ & 1.0 & 70 \\
\hline 11 & DMA & $\mathrm{Ni}(C O D)_{2}$ & 1.3 & 87 \\
\hline 12 & DMA & $\mathrm{Ni}(C O D)_{2}$ & 2.0 & 86 \\
\hline
\end{tabular}


Table 2 Substrate scope with monocyclic anhydrides and alkyl bromidesa.

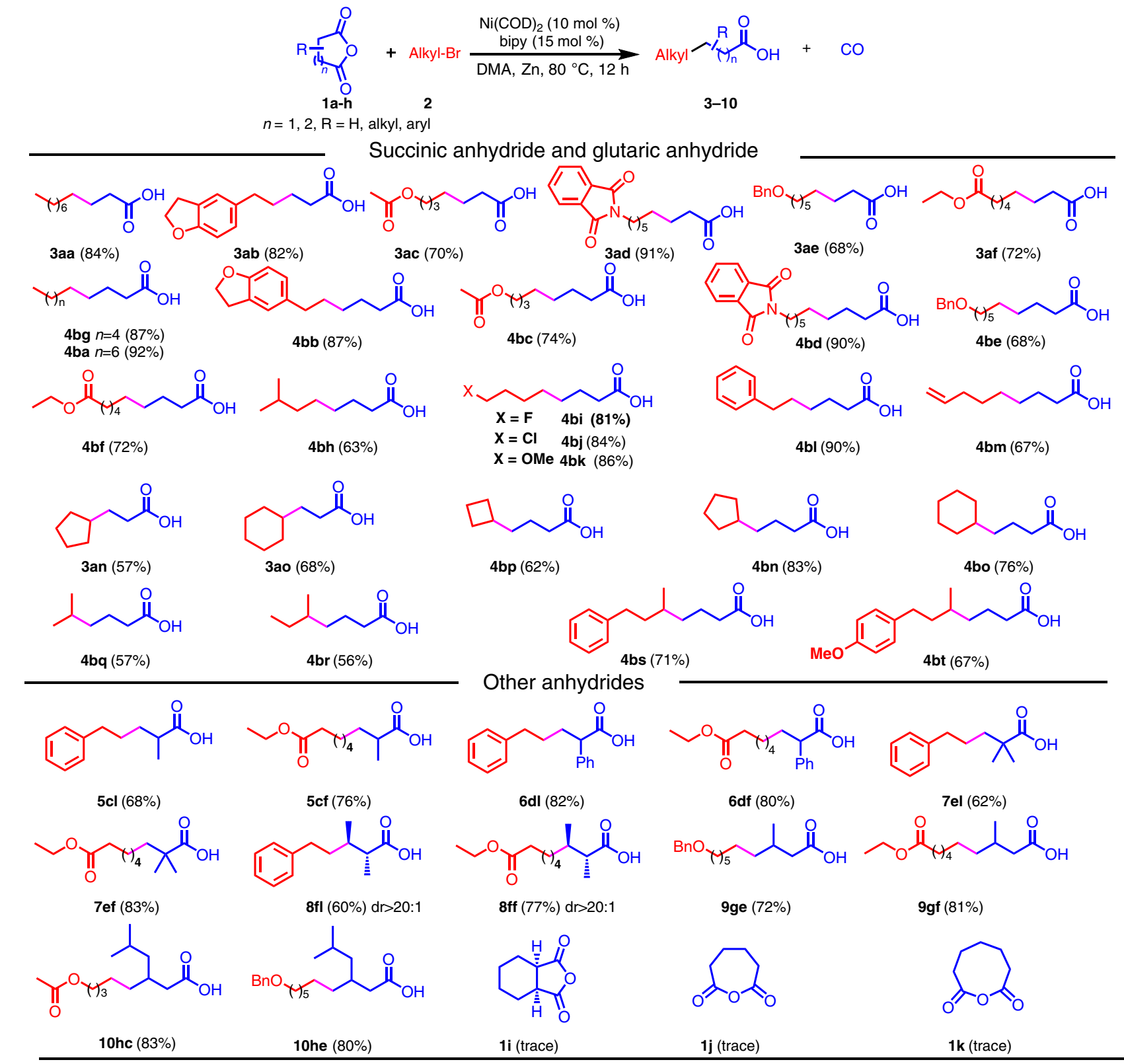

aReactions performed on a $0.3 \mathrm{mmol}$ scale with 1.5 equiv anhydride, 1.0 equiv alkyl bromide, 2 equiv zinc powder in $0.225 \mathrm{~mL}$ DMA.

68-92\% yields (4ba-4bg). $\beta$-Branched alkyl bromides are expected to generate nickel alkyl intermediates that tend to undergo relatively fast $\beta$-hydride elimination ${ }^{56,57}$. Nonetheless, isobutyl bromide was compatible under the standard conditions, furnishing $4 \mathrm{bh}$ in $63 \%$ yield. Furthermore, alkyl bromides bearing halogens, such as fluoro and chloro reacted with excellent chemoselectivity, affording the corresponding products in 81 and $84 \%$ yield, respectively. Alkyl bromides containing ether, homobenzyl, and double bonds reacted efficiently, affording the products in $67-90 \%$ yields.

In addition to primary alkyl bromides, a variety of secondary alkyl bromides were compatible with the standard conditions. In general, both cyclic and acyclic secondary alkyl bromides, such as cyclopentyl-, cyclohexyl-, cyclobutyl-, isopropyl-, and sec-butyl- bromide, exhibited good reactivity, affording $\beta$ - and $\gamma$-functionalized propionic and butyric acids, respectively (3an, 3ao, and 4bn-4bt, 56-83\%). Unfortunately, when tertiary bromides were subjected to coupling with anhydrides, no products were obtained under the standard conditions.

We next explored the scope of the unsymmetrical anhydrides. It is observed that unsymmetrical succinic anhydrides such as 2methylsuccinic anhydride 1c, 2-phenylsuccinic anhydride 1d and 2,2-dimethylsuccinic anhydride $\mathbf{1 e}$ could couple with $\mathbf{2 1}$ and $\mathbf{2 f}$ affording $\beta$-functionalized acids in $68-83 \%$ yield. It is noteworthy that the oxidative addition and decarbonylation processes took place at the less sterically hindered side of the anhydride. Disubstituted anhydrides, such as cis-2,3-dimethylsuccinic anhydride $\mathbf{1 f}$ is a competent coupling partner, affording $\mathbf{8 f l}$ and $\mathbf{8 f f}$ in $60 \%$ and $77 \%$ yield, respectively. It should be noted that $1 \mathrm{f}$ led to the corresponding products with high stereochemical fidelity 


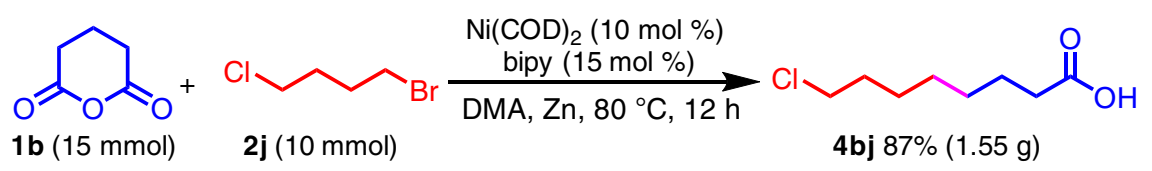

Fig. 3 Scale up the transformation to $\mathbf{1 0 . 0} \mathbf{~ m m o l}$. 1-Bromo-4-chlorobutane reacted with $\mathbf{1 b}$ to give the desired product $\mathbf{4 b j}$ with $87 \%$ yield.

a

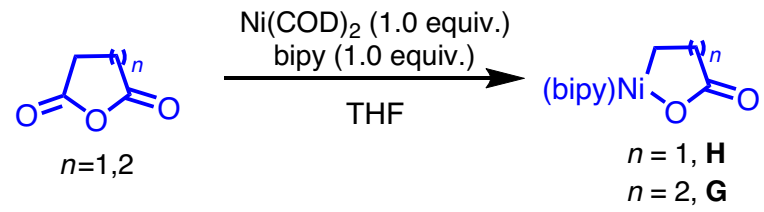

b<smiles>O=C1CC[NH+]([O-])O1</smiles>

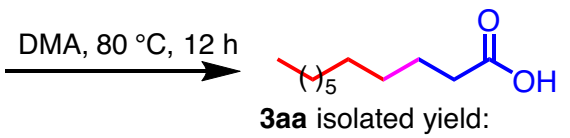

3aa isolated yield:

H

2a

$72 \%$ (standard conditions)

$68 \%$ (in the absence of $\mathrm{Zn}$ )
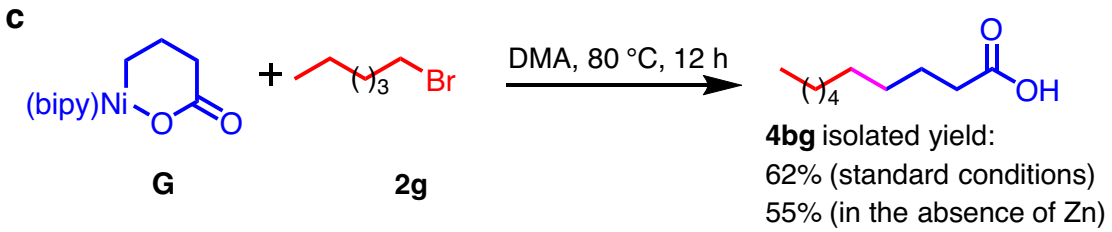

Fig. 4 Preliminary mechanistic studies. a Preparation of Ni-homoenolate and its higher homolog. b Alkylation of the independently synthesized homoenolate $\mathbf{H}$. c Alkylation of the independently synthesized $\mathbf{G}$ with alkyl bromides.

$(>20: 1$ dr). Furthermore, methyl and isobutyl substituted glutaric anhydrides $\mathbf{1 g}$ and $\mathbf{1 h}$ afforded $\gamma$-cross-coupled products in $72-83 \%$ yield. Unfortunately, bicyclic anhydrides, such as cis1,2-cyclohexanedicarboxylic anhydride $\mathbf{1 i}$ and larger ring-sized anhydrides $(\mathbf{1} \mathbf{j}$ and $\mathbf{1 k})$, gave only trace coupling products under the standard conditions.

To test the scalability of this transformation, $15.0 \mathrm{mmol}$ of glutaric anhydride $\mathbf{1 b}$ was coupled with $10.0 \mathrm{mmol}$ of 1-bromo-4chlorobutane $\mathbf{2} \mathbf{j}$ under the standard conditions. The decarbonylative cross-coupled product $\mathbf{4 b j}$ was isolated in $87 \%$ yield $(1.55 \mathrm{~g})$ (Fig. 3).

Mechanistic studies. Preliminary mechanistic studies demonstrated that nickel homoenolates are intermediates in this catalytic system. Ni-homoenolate $\mathbf{H}$ and its higher homolog $\mathbf{G}$ were synthesized independently ${ }^{58,59}$ (Fig. 4a). Exposure of $\mathbf{H}$ or $\mathbf{G}$ to alkyl bromides in the presence or absence of reducing agent generated 3aa and $\mathbf{4 b g}$ in good yields (Fig. 4b, c). These results demonstrate that Ni-bound homoenolate $\mathbf{H}$ and its derivative $\mathbf{G}$ are viable intermediates in these transformations. We have ruled out the possibility of cyclic anhydrides undergoing $\mathrm{CO}$ deinsertion followed by $\beta$-hydride elimination to generate acrylate intermediates and conjugate addition of radicals to these intermediates (see Supplementary Information). Notably, the stoichiometric reactions were successful in the absence of zinc powder (the source of electrons for this process may be the $\mathrm{Ni}(\mathrm{I})$ product reducing the alkyl bromide; see the catalytic cycle discussed below) ${ }^{60,61}$. These results lead to the hypothesis that zinc powder acts as a reductant to nickel to make these reactions catalytic $^{62-67}$.

To explore the selectivity in the oxidative addition to (L) $\mathrm{Ni}^{0}$, we examined the relative reactivities of glutaric anhydride (1b) and 1-bromohexane (2g) using stoichiometric $\mathrm{Ni}(\mathrm{COD})_{2}$ and bipy (Table 3) in a study that was inspired by the Weix group's report of oxidative addition with nickel using aryl and alkyl halides ${ }^{68}$. After these reagents were stirred at room temperature for several hours, the reaction was quenched with $1 \mathrm{M} \mathrm{HCl}$. The relative reactivities were determined by the loss of each starting material and formation of $\mathbf{G}$ and $\mathbf{4} \mathbf{b g}^{68}$ (Table 3 ). We found anhydride $\mathbf{1 b}$ was consumed much faster than alkyl bromide $\mathbf{2} \mathbf{~ g}$. These data indicate that the anhydride undergoes oxidative addition faster than the alkyl bromide in the presence of $\mathrm{Ni}$ $(\mathrm{COD})_{2}$ and bipy, and that $\mathbf{G}$ is a likely intermediate in the catalytic reaction.

To further probe the reaction mechanism, radical trap and radical clock experiments were employed (Fig. 5a, b) ${ }^{66,69}$. Under otherwise standard conditions with alkyl bromide $2 \mathbf{l}$, when the radical scavenger TEMPO was added, product formation was suppressed. If the alkyl bromide is activated through a radical mechanism, ring-opened products would be expected with cyclopropylmethyl bromide (2u). In the event, $\mathbf{2 u}$ led primarily to unsaturated acid 4bu' (52\% AY), arising from rapid ring opening of the cyclopropyl-methyl radical to the homoallylic radical.

Inspired by Weix's 5-exo-trig experiment to evaluate ringclosed vs. ring-opened ratios as a function of catalyst loading68, we chose glutaric anhydride (1b) and 5-hexenyl bromide (2v) to examine the ratio of decarbonylative product $\mathbf{4 b v}$ and cyclized product $\mathbf{4 b v}$ '. By changing the catalyst loading of $\mathrm{Ni}(\mathrm{COD})_{2}$ from $5 \mathrm{~mol} \%$ to $40 \mathrm{~mol} \%$, the ratio of $\mathbf{4 b v} / \mathbf{4 b v}$ was observed to increase linearly (Fig. 5c).

Finally, we probed the possibility of an organozinc intermediate in this cross-coupling, potentially formed from reaction of $\mathrm{Zn}^{0}$ with the alkyl bromide. Thus, we independently synthesized organozinc reagent $\mathbf{J}$ and subjected it to the metallocycle $\mathbf{G}$ under catalytically relevant conditions (Fig. 5d). No conversion to cross-coupled product was observed. Taken 
Table 3 Selectivity in oxidative addition to (L)NiOa.

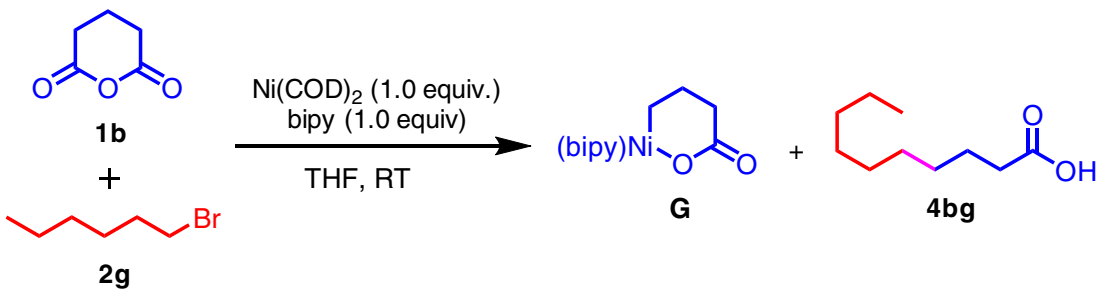

\begin{tabular}{lllll}
\hline Entry & $\mathbf{t}(\mathbf{h})$ & $\mathbf{G ~ ( \% ~ A Y ) ~}$ & $\mathbf{2 g}(\% \mathbf{A Y})^{\mathbf{b}, \mathbf{c}}$ & 4bg (\% AY) \\
\hline 1 & 1 & 68 & 97 & trace \\
2 & 2 & 72 & 95 & trace \\
3 & 3 & 76 & 95 & trace \\
\hline
\end{tabular}

aA 1:1 mixture of $\mathbf{1 a}: \mathbf{2 g}$ was added to a THF solution of 1.0 equiv. Ni(COD $)_{2}$ and 1.0 equiv. bipy.

a

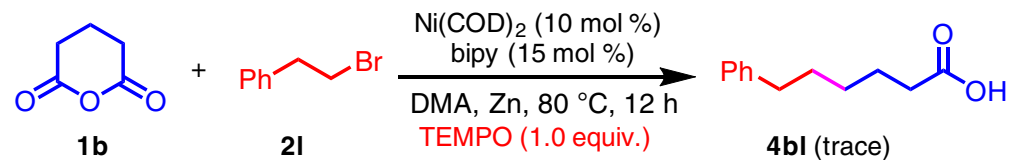

b

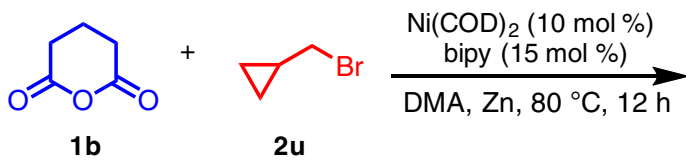<smiles>C=CCCCCCC(=O)O</smiles>

4 bu' $^{\prime}(52 \%)$

C<smiles>O=C1CCCC(=O)O1</smiles>

$1 \mathrm{~b}$ 2v

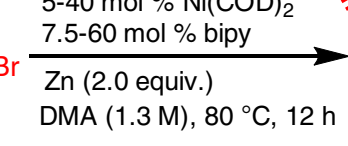<smiles>C=CCCCCCCCC(=O)O</smiles>

$4 b^{\prime}$

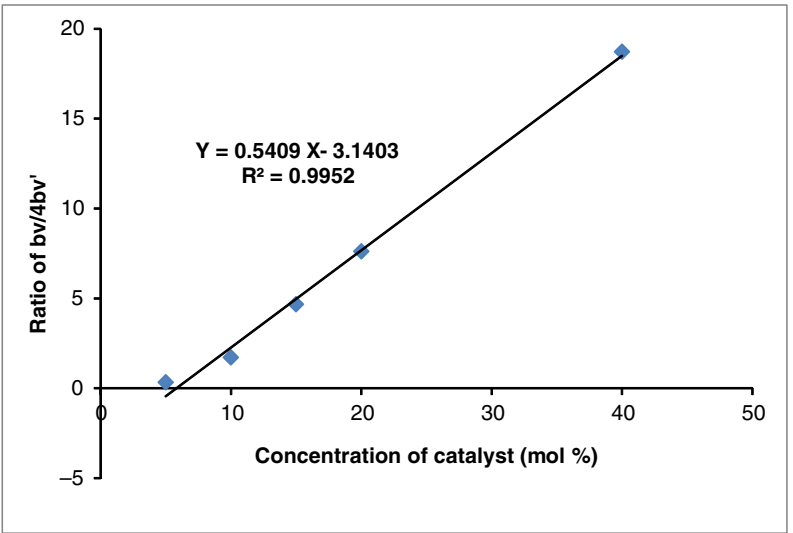

d

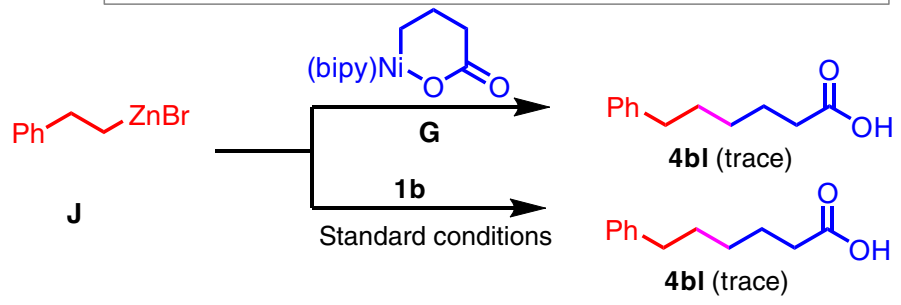

Fig. 5 Mechanistic studies. a Inhibition of the reaction in the presence of TEMPO. b Radical clock reaction leads to ring opening, suggestive of radical intermediates. c Dependence of the ratio of $\mathbf{4 b v / 4 b v ' ~ o n ~ c a t a l y s t ~ c o n c e n t r a t i o n , ~ s u p p o r t i n g ~ a ~ r a d i c a l ~ m e c h a n i s m . ~} \mathbf{d}$ Demonstration that organozinc $\mathbf{J}$ is not a viable reaction intermediate. 


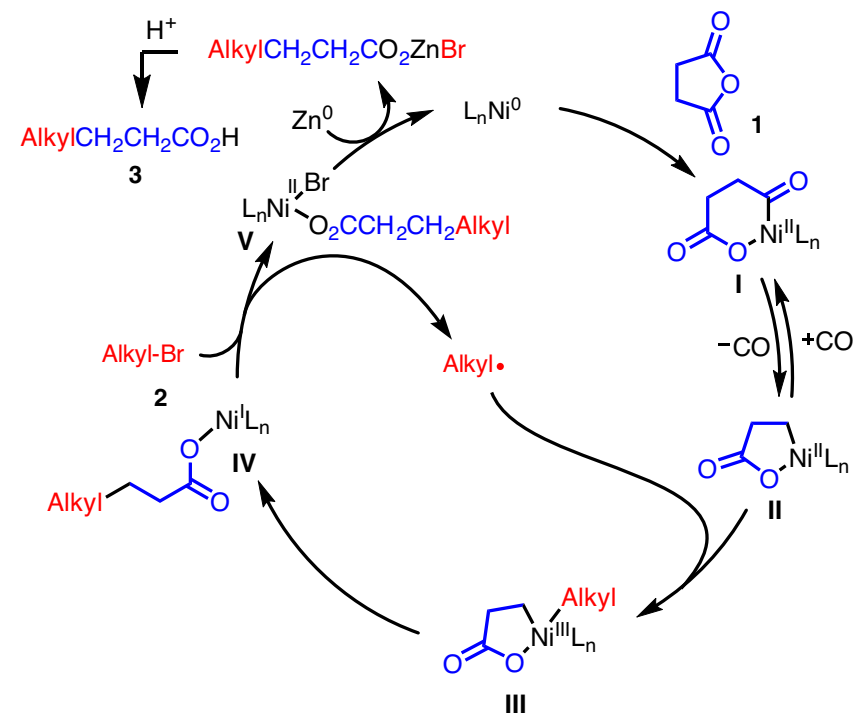

Fig. 6 Mechanistic hypothesis. Oxidative addition of anhydride (1) is followed by loss of carbon monoxide to generate homoenolate II. Oxidative capture of the alkyl radical formed $\mathrm{Ni}(\mathrm{III})$, which reductively eliminates to form $\mathrm{Ni}(\mathrm{I})$ intermediate IV. The $\mathrm{Ni}(\mathrm{I})$ intermediate is proposed to reduce the alkyl bromide $\mathbf{2}$ to furnish the alkyl radical and $\mathrm{Ni}(\mathrm{II})$ intermediate V. Zinc reduction of $\mathrm{Ni}(\mathrm{II})$ reforms $\mathrm{Ni}(\mathrm{O})$ and produces a zinc carboxylate, closing the catalytic cycle. Upon workup, the zinc carboxylate is protonated to give the observed carboxylic acid product $\mathbf{3}$.

together, these experiments support single electron transfer (SET) processes $^{70}$ and discount an organozinc intermediate.

Grounded in the experimental results presented above, a plausible mechanism is proposed (Fig. 6). $\mathrm{Ni}^{0}$ oxidatively adds cyclic anhydride 1 giving a $\mathrm{Ni}^{\mathrm{II}}$ species $(\mathbf{I})^{71-74}$. Next, decarbonylation of I with loss of $\mathrm{CO}$ forms the primary $\mathrm{C}\left(\mathrm{sp}^{3}\right)$ $\mathrm{Ni}$ bond ${ }^{75}$ in II. The next step is generation of the alkyl radical via SET. This could take place from $\mathrm{Ni}(0)$ or $\mathrm{Ni}(\mathrm{I})$. We postulate that an intermediate $\mathrm{Ni}(\mathrm{I})$ performs the SET, because Table 3 suggests that reaction of $\mathrm{Ni}(0)$ with alkyl bromide is slow relative to oxidative addition of the anhydride. The alkyl radical is oxidatively trapped to provide the $\mathrm{Ni}^{\mathrm{III}}$ complex (III). Reductive elimination of intermediate III generates the $\mathrm{C}-\mathrm{C}$ bond as well as the reactive $\mathrm{Ni}^{\mathrm{I}}$ carboxylate species IV. As noted above, this $\mathrm{Ni}(\mathrm{I})$ species is proposed to reduce the alkyl bromide via SET giving BrNi(II)(carboxylate) (V) and an alkyl radical. Finally, the $\mathrm{Ni}^{\mathrm{II}}$ product $\mathrm{V}$ is reduced by $\mathrm{Zn}^{0}$ to regenerate $\mathrm{Ni}^{0}$ and the zinc carboxylate. Acidic workup liberates the acid coupling product, 3.

\section{Discussion}

We have developed a nickel-catalyzed generation of homoenolates and their higher homologs via decarbonylation of monocyclic anhydrides. The in situ-formed nickel homoenolate derivatives were successfully coupled with unactivated alkyl bromides, generating a diverse array of functionalized aliphatic acids. Preliminary mechanistic studies demonstrated that the nickel(0) catalyst selectively reacts with anhydrides over alkyl bromides leading to the key homoenolates. Radical clock and related studies indicate that organozinc intermediates are unlikely and point to the involvement of alkyl radical species. Key advantages of this method include excellent functional group compatibility, mild reaction conditions, and avoidance of prefunctionalized organometallic reagents. This method enables construction of valuable carboxylic acids. Further studies using anhydrides as homoenolate precursors are ongoing in our team.

\begin{abstract}
Methods
General procedure for the synthesis of aliphatic acids. To an oven-dried microwave vial $(10 \mathrm{~mL})$ equipped with a stir bar $(10 \times 5 \mathrm{~mm})$ was added $\mathrm{Ni}(\mathrm{COD})_{2}$ $(8.3 \mathrm{mg}, 0.03 \mathrm{mmol})$ and bipy $(7.0 \mathrm{mg}, 0.045 \mathrm{mmol})$ under an argon atmosphere inside a glove box at $25^{\circ} \mathrm{C}$. Next, $0.225 \mathrm{~mL}$ of dry DMA was added via syringe to give a purple solution. After the catalyst/ligand solution was stirred for $1 \mathrm{~h}$ at $25^{\circ} \mathrm{C}$ inside the glove box, $\mathrm{Zn}$ powder $(39.2 \mathrm{mg}, 0.6 \mathrm{mmol}, 2.0$ equiv) was added to the reaction vial followed by the monocyclic anhydride $(0.45 \mathrm{mmol}, 1.5$ equiv) and alkyl bromide $(0.3 \mathrm{mmol}, 1.0$ equiv). The microwave vial was sealed with a cap containing a rubber septum and removed from the glove box. The reaction mixture was stirred ( $\sim 1000$ r.p.m.) at $80^{\circ} \mathrm{C}$ for $12 \mathrm{~h}$. The resulting gray solution was cooled to room temperature, quenched by the addition of five drops of water via syringe through the septum and then the vial opened to air. The reaction mixture was passed through a short flash column chromatography in silica gel (200-300 mesh) and rinsed with $5 \mathrm{~mL}$ of ethyl acetate to afford a yellow solution. The solvent and volatile materials were removed by rotary evaporator. The crude residue was purified by flash column chromatography in silica gel to yield the corresponding product.
\end{abstract}

\section{Data availability}

Detailed experimental procedures and characterization of compounds can be found in the Supplementary Information. The authors declare that all other data supporting the findings of this study are available within the article and Supplementary Information files, and also are available from the corresponding authors.

Received: 20 April 2020; Accepted: 22 September 2020; Published online: 06 November 2020

\section{References}

1. Mills, L. R. \& Rousseaux, S. A. L. Modern developments in the chemistry of homoenolates. Eur. J. Org. Chem. 2019, 8-26 (2019).

2. Murauski, K. J. R., Jaworski, A. A. \& Scheidt, K. A. A continuing challenge: Nheterocyclic carbene-catalyzed syntheses of $\gamma$-butyrolactones. Chem. Soc. Rev. 47, 1773-1782 (2018).

3. Rosa, D., Nikolaev, A., Nithiy, N. \& Orellana, A. Palladium-catalyzed crosscoupling reactions of cyclopropanols. Synlett 26, 441-448 (2015).

4. Menon, R. S., Biju, A. T. \& Nair, V. Recent advances in employing homoenolates generated by $\mathrm{N}$-heterocyclic carbene (NHC) catalysis in carbon-carbon bond-forming reactions. Chem. Soc. Rev. 44, 5040-5052 (2015).

5. Hopkinson, M. N., Richter, C., Schedler, M. \& Glorius, F. An overview of Nheterocyclic carbenes. Nature 510, 485-496 (2014).

6. Grossmann, A. \& Enders, D. N-heterocyclic carbene catalyzed domino reactions. Angew. Chem., Int. Ed. 51, 314-325 (2012).

7. Nithiy, N., Rosa, D. \& Orellana, A. Carbon-carbon bond formation through palladium homoenolates. Synthesis 45, 3199-3210 (2013).

8. Nair, V., Vellalath, S. \& Babu, B. P. Recent advances in carbon-carbon bondforming reactions involving homoenolates generated by NHC catalysis. Chem Soc. Rev. 37, 2691-2698 (2008).

9. Nickon, A. \& Lambert, J. L. Homoenolate anions. J. Am. Chem. Soc. 84, 4604-4605 (1962).

10. Nakamura, E. \& Kuwajima, I. Homoenolate anion precursor. Reaction of ester homoenol silyl ether with carbonyl compounds. J. Am. Chem. Soc. 99, 7360-7362 (1977).

11. Seppi, M., Kalkofen, R., Reupohl, J., Froehlich, R. \& Hoppe, D. Highly enantiomerically enriched ketone homoenolate reagents prepared by (-)-sparteine-mediated $\gamma$-deprotonation of achiral 1 -alkenyl carbamates. Angew. Chem. Int. Ed. 43, 1423-1427 (2004).

12. Pippel, D. J., Curtis, M. D., Du, H. \& Beak, P. Complex-induced proximity effects: stereoselective carbon-carbon bond formation in chiral auxiliary mediated $\beta$-lithiation-substitution sequences of $\beta$-substituted secondary carboxamides. J. Org. Chem. 63, 2-3 (1998).

13. Beak, P., Hunter, J. E. \& Jun, Y. M. Dominance of the proximity effect of complexation over resonance and inductive effects in directing a metalation: regiospecific $\beta$ lithiation of N,N-diisopropylcyclohex-3-enecarboxamide and of N,N-diisopropyl-2-methylpent-3-enecarboxamide. J. Am. Chem. Soc. 105, 6350-6351 (1983).

14. Mills, L. R., Barrera Arbelaez, L. M. \& Rousseaux, S. A. L. Electrophilic zinc homoenolates: synthesis of cyclopropylamines from cyclopropanols and amines. J. Am. Chem. Soc. 139, 11357-11360 (2017).

15. Aspin, S., Goutierre, A.-S., Larini, P., Jazzar, R. \& Baudoin, O. Synthesis of aromatic a-aminoesters: palladium-catalyzed long-range arylation of primary $\mathrm{C}_{\mathrm{sp} 3}-\mathrm{H}$ bonds. Angew. Chem. Int. Ed. 51, 10808-10811 (2012).

16. Renaudat, A. et al. Palladium-catalyzed $\beta$-arylation of carboxylic esters. Angew. Chem. Int. Ed. 49, 7261-7265 (2010). 
17. Shen, Z.-L. et al. Synthesis of water-tolerant indium homoenolate in aqueous media and its application in the synthesis of 1,4-dicarbonyl compounds via palladium-catalyzed coupling with acid chloride. J. Am. Chem. Soc. 132, 15852-15855 (2010).

18. Nomura, K. \& Matsubara, S. Preparation of zinc-homoenolate from asulfonyloxy ketone and bis(iodozincio)methane. Chem. Lett. 36, 164-165 (2007).

19. Yeh, M. C. P. \& Knochel, P. 2-Cyanoethylzinc iodide: a new reagent with reactivity umpolung. Tetrahedron Lett. 29, 2395-2396 (1988).

20. Tamaru, Y., Ochiai, H., Nakamura, T. \& Yoshida, Z. Synthesis of $\beta, \gamma, \delta-, \varepsilon-$, and $\zeta$-zinc ketones and their transition metal catalyzed reaction with carbon electrophiles. Angew. Chem. Int. Ed. 26, 1157-1158 (1987).

21. Park, H. S., Fan, Z., Zhu, R.-Y. \& Yu, J.-Q. Distal $\gamma$-C $\left(\mathrm{sp}^{3}\right)-\mathrm{H}$ olefination of ketone derivatives and free carboxylic acids. Angew. Chem. Int. Ed. 59, 12853-12859 (2020).

22. Aoki, S., Fujimura, T., Nakamura, E. \& Kuwajima, I. Palladium-catalyzed arylation of siloxycyclopropanes with aryl triflates. Carbon chain elongation via catalytic carbon-carbon bond cleavage. J. Am. Chem. Soc. 110, 3296-3298 (1988).

23. Rosa, D. \& Orellana, A. Palladium-catalyzed cross-coupling of cyclopropanolderived ketone homoenolates with aryl bromides. Chem. Commun. 49, 5420-5422 (2013).

24. Rosa, D. \& Orellana, A. Palladium-catalyzed cross-coupling of cyclopropanols with aryl halides under mild conditions. Org. Lett. 13, 110-113 (2011).

25. Parida, B. B., Das, P. P., Niocel, M. \& Cha, J. K. C-acylation of cyclopropanols: preparation of functionalized 1,4-diketones. Org. Lett. 15, 1780-1783 (2013).

26. Nithiy, N. \& Orellana, A. Palladium-catalyzed cross-coupling of benzyl chlorides with cyclopropanol-derived ketone homoenolates. Org. Lett. 16, 5854-5857 (2014).

27. Wu, P., Jia, M., Lin, W. \& Ma, S. Matched coupling of propargylic carbonates with cyclopropanols. Org. Lett. 20, 554-557 (2018).

28. Wright, T. B., Turnbull, B. W. H. \& Evans, P. A. Enantioselective rhodiumcatalyzed allylic alkylation of $\beta, \gamma$-unsaturated $\alpha$-amino nitriles: synthetic homoenolate equivalents. Angew. Chem. Int. Ed. 58, 9886-9890 (2019).

29. Ye, Z., Cai, X., Li, J. \& Dai, M. Catalytic cyclopropanol ring opening for divergent syntheses of $\gamma$-butyrolactones and $\delta$-ketoesters containing allcarbon quaternary centers. ACS Catal. 8, 5907-5914 (2018).

30. Ye, Z., Gettys, K. E., Shen, X. \& Dai, M. Copper-catalyzed cyclopropanol ring opening $\mathrm{C}_{\mathrm{sp} 3}-\mathrm{C}_{\mathrm{sp} 3}$ cross-couplings with (fluoro)alkyl halides. Org. Lett. 17, 6074-6077 (2015).

31. Das, P. P., Belmore, K. \& Cha, J. K. $\mathrm{S}_{\mathrm{N} 2^{\prime}}$ alkylation of cyclopropanols via homoenolates. Angew. Chem. Int. Ed. 51, 9517-9520 (2012).

32. Huo, H., Gorsline, B. J. \& Fu, G. C. Catalyst-controlled doubly enantioconvergent coupling of racemic alkyl nucleophiles and electrophiles. Science 367, 559-564 (2020).

33. Uttry, A. \& van Gemmeren, M. Direct $\mathrm{C}\left(\mathrm{sp}^{3}\right)$ - $\mathrm{H}$ activation of carboxylic acids. Synthesis 52, 479-488 (2020).

34. Le, K. K. A., Nguyen, H. \& Daugulis, O. 1-Aminopyridinium Ylides as monodentate directing groups for $\mathrm{sp}^{3} \mathrm{C}-\mathrm{H}$ bond functionalization. J. Am. Chem. Soc. 141, 14728-14735 (2019).

35. Zhuang, Z. et al. Ligand-enabled $\beta-\mathrm{C}\left(\mathrm{sp}^{3}\right)-\mathrm{H}$ olefination of free carboxylic acids. J. Am. Chem. Soc. 140, 10363-10367 (2018).

36. Shabashov, D. \& Daugulis, O. Auxiliary-assisted palladium-catalyzed arylation and alkylation of $\mathrm{sp}^{2}$ and $\mathrm{sp}^{3}$ carbon-hydrogen bonds. J. Am. Chem. Soc. 132, 3965-3972 (2010).

37. Ghosh, K. K. et al. Ligand-enabled $\gamma-\mathrm{C}\left(\mathrm{sp}^{3}\right)-\mathrm{H}$ olefination of free carboxylic acids. Angew. Chem. Int. Ed. 59, 12848-12852 (2020).

38. Wu, X., Zhao, Y. \& Ge, H. Nickel-catalyzed site-selective alkylation of unactivated $\mathrm{C}\left(\mathrm{sp}^{3}\right)-\mathrm{H}$ bonds. J. Am. Chem. Soc. 136, 1789-1792 (2014).

39. Chatani, N. Nickel-catalyzed C-H bond functionalization utilizing an N,N'bidentate directing group. Top. Organomet. Chem. 56, 19-46 (2016).

40. Mills, L. R., Zhou, C., Fung, E. \& Rousseaux, S. A. L. Ni-catalyzed $\beta$-alkylation of cyclopropanol-derived homoenolates. Org. Lett. 21, 8805-8809 (2019).

41. Iwasaki, T. \& Kambe, N. Ni-catalyzed C-C couplings using alkyl electrophiles. Top. Curr. Chem. 374, 1-36 (2016).

42. Rudolph, A. \& Lautens, M. Secondary alkyl halides in transition-metalcatalyzed cross-coupling reactions. Angew. Chem. Int. Ed. 48, 2656-2670 (2009).

43. Frisch, A. C. \& Beller, M. Catalysts for cross-coupling reactions with nonactivated alkyl halides. Angew. Chem. Int. Ed. 44, 674-688 (2005).

44. Cherney, A. H., Kadunce, N. T. \& Reisman, S. E. Enantioselective and enantiospecific transition-metal-catalyzed cross-coupling reactions of organometallic reagents to construct C-C bonds. Chem. Rev. 115, 9587-9652 (2015).

45. Yang, W. et al. Synergistic N-heterocyclic carbene/palladium-catalyzed umpolung 1,4-addition of aryl iodides to enals. Angew. Chem. Int. Ed. 59, 161-166 (2020).

46. Cheng, K. \& Walsh, P. J. Arylation of aldehyde homoenolates with aryl bromides. Org. Lett. 15, 2298-2301 (2013).
47. Lin, T. et al. Nickel-catalyzed desymmetrizing cross-electrophile coupling of cyclic meso-anhydrides. Org. Lett. 20, 1191-1194 (2018)

48. O’Brien, E. M., Bercot, E. A. \& Rovis, T. Decarbonylative cross-coupling of cyclic anhydrides: introducing stereochemistry at an $\mathrm{sp}^{3}$ carbon in the crosscoupling event. J. Am. Chem. Soc. 125, 10498-10499 (2003).

49. Yamamoto, T., Sano, K. \& Yamamoto, A. Effect of ligand on ring contraction of six-membered nickel-containing cyclic esters, $\mathrm{L}_{\mathrm{n}} \mathrm{NiCH}_{2} \mathrm{CH}_{2} \mathrm{CH}_{2} \mathrm{COO}$, to their five-membered-ring isomers, $\mathrm{L}_{\mathrm{n}} \mathrm{NiCH}\left(\mathrm{CH}_{3}\right) \mathrm{CH}_{2} \mathrm{COO}$. Kinetic and thermodynamic control of asymmetric induction by chiral diphosphines in the ring contraction. J. Am. Chem. Soc. 109, 1092-1100 (1987).

50. Julia-Hernandez, F., Moragas, T., Cornella, J. \& Martin, R. Remote carboxylation of halogenated aliphatic hydrocarbons with carbon dioxide. Nature 545, 84-88 (2017).

51. Borjesson, M., Moragas, T. \& Martin, R. Ni-catalyzed carboxylation of unactivated alkyl chlorides with $\mathrm{CO}_{2}$. J. Am. Chem. Soc. 138, 7504-7507 (2016).

52. Landoni, M. F. \& Soraci, A. Pharmacology of chiral compounds: 2arylpropionic acid derivatives. Curr. Drug Metab. 2, 37-51 (2001).

53. Rodriguez, N. \& Goossen, L. J. Decarboxylative coupling reactions: a modern strategy for C-C-bond formation. Chem. Soc. Rev. 40, 5030-5048 (2011).

54. Goossen, L. J., Rodriguez, N. \& Goossen, K. Carboxylic acids as substrates in homogeneous catalysis. Angew. Chem. Int. Ed. 47, 3100-3120 (2008).

55. Stache, E. E., Ertel, A. B., Rovis, T. \& Doyle, A. G. Generation of phosphoranyl radicals via photoredox catalysis enables voltage independent activation of strong C-O bonds. ACS Catal. 8, 11134-11139 (2018).

56. Yan, X.-B., Li, C.-L., Jin, W.-J., Guo, P. \& Shu, X.-Z. Reductive coupling of benzyl oxalates with highly functionalized alkyl bromides by nickel catalysis. Chem. Sci. 9, 4529-4534 (2018).

57. Yonova, I. M. et al. Stereospecific nickel-catalyzed cross-coupling reactions of alkyl Grignard reagents and identification of selective anti-breast-cancer agents. Angew. Chem., Int. Ed. 53, 2422-2427 (2014).

58. Castan o, A. M. \& Echavarren, A. M. Synthesis of protected 3-methylaspartic acids from glutamic anhydride via nickelacycles. Tetrahedron Lett. 34, 4361-4362 (1993)

59. Sano, K., Yamamoto, T. \& Yamamoto, A. Preparation of Ni- or Pt-containing cyclic esters by oxidative addition of cyclic carboxylic anhydrides and their properties. Bull. Chem. Soc. Jpn. 57, 2741-2747 (1984).

60. Hegedus, L. S. \& Thompson, D. H. P. The reactions of organic halides with ( $\pi$ allyl)nickel halide complexes: a mechanistic study. J. Am. Chem. Soc. 107, 5663-5669 (1985)

61. Hegedus, L. S. \& Miller, L. L. Reaction of $\pi$-allylnickel bromide complexes with organic halides. Stereochemistry and mechanism. J. Am. Chem. Soc. 97, 459-460 (1975).

62. Ye, Y., Chen, H., Sessler, J. L. \& Gong, H. Zn-mediated fragmentation of tertiary alkyl oxalates enabling formation of alkylated and arylated quaternary carbon centers. J. Am. Chem. Soc. 141, 820-824 (2019).

63. Wang, J., Cary, B. P., Beyer, P. D., Gellman, S. H. \& Weix, D. J. Ketones from nickel-catalyzed decarboxylative, non-symmetric cross-electrophile coupling of carboxylic acid esters. Angew. Chem. Int. Ed. 58, 12081-12085 (2019).

64. Chen, F. et al. Remote migratory cross-electrophile coupling and olefin hydroarylation reactions enabled by in situ generation of NiH. J. Am. Chem. Soc. 139, 13929-13935 (2017)

65. Huihui, K. M. M. et al. Decarboxylative cross-electrophile coupling of Nhydroxyphthalimide esters with aryl iodides. J. Am. Chem. Soc. 138, 5016-5019 (2016)

66. Zhao, C., Jia, X., Wang, X. \& Gong, H. Ni-catalyzed reductive coupling of alkyl acids with unactivated tertiary alkyl and glycosyl halides. J. Am. Chem. Soc. 136, 17645-17651 (2014)

67. Cherney, A. H. \& Reisman, S. E. Nickel-catalyzed asymmetric reductive crosscoupling between vinyl and benzyl electrophiles. J. Am. Chem. Soc. 136, 14365-14368 (2014)

68. Biswas, S. \& Weix, D. J. Mechanism and selectivity in nickel-catalyzed crosselectrophile coupling of aryl halides with alkyl halides. J. Am. Chem. Soc. 135, 16192-16197 (2013).

69. Huang, L., Olivares, A. M. \& Weix, D. J. Reductive decarboxylative alkynylation of N-hydroxyphthalimide esters with bromoalkynes. Angew. Chem. Int. Ed. 56, 11901-11905 (2017)

70. Correa, A., Leon, T. \& Martin, R. Ni-catalyzed carboxylation of $\mathrm{C}\left(\mathrm{sp}^{2}\right)$ - and C $\left(\mathrm{sp}^{3}\right)-\mathrm{O}$ bonds with $\mathrm{CO}_{2}$. J. Am. Chem. Soc. 136, 1062-1069 (2014)

71. Diccianni, J., Lin, Q. \& Diao, T. Mechanisms of nickel-catalyzed coupling reactions and applications in alkene functionalization. Acc. Chem. Res. 53, 906-919 (2020).

72. Diccianni, J. \& Diao, T. Mechanisms of nickel-catalyzed cross-coupling reactions. Trends Chem. 1, 830-844 (2019).

73. Johnson, J. B., Bercot, E. A., Rowley, J. M., Coates, G. W. \& Rovis, T. Ligand dependent catalytic cycle and role of styrene in nickel-catalyzed anhydride cross-coupling: evidence for turnover-limiting reductive elimination. J. Am. Chem. Soc. 129, 2718-2725 (2007). 
74. Bercot, E. A. \& Rovis, T. A mild and efficient catalytic alkylative monofunctionalization of cyclic anhydrides. J. Am. Chem. Soc. 124, 174-175 (2002).

75. Guo, L. \& Rueping, M. Decarbonylative cross-couplings: nickel catalyzed functional group interconversion strategies for the construction of complex organic molecules. Acc. Chem. Res. 51, 1185-1195 (2018).

\section{Acknowledgements}

We acknowledge the National Natural Science Foundation of China (21801128 and 22071107 to J.M.), Natural Science Foundation of Jiangsu Province, China (BK20170965 to J.M.), and Nanjing Tech University (39837112) for financial support. P.J.W. thanks the US National Science Foundation (CHE-1902509).

\section{Author contributions}

T.L. performed most of the experiments and mechanistic study with the help of Y.G., P.Q., and H.G. The project conceived J.M. and T.L. with help from P.J.W. The project was directed by J.M. and the manuscript was written by T.L., J.M., and P.J.W.

\section{Competing interests}

The authors declare no competing interests.

\section{Additional information}

Supplementary information is available for this paper at https://doi.org/10.1038/s41467020-19194-x.
Correspondence and requests for materials should be addressed to P.J.W. or J.M.

Peer review information Nature Communications thanks Naohiko Yoshikai and the other, anonymous, reviewer(s) for their contribution to the peer review of this work. Peer reviewer reports are available.

Reprints and permission information is available at http://www.nature.com/reprints

Publisher's note Springer Nature remains neutral with regard to jurisdictional claims in published maps and institutional affiliations.

\section{(c) (i)}

Open Access This article is licensed under a Creative Commons Attribution 4.0 International License, which permits use, sharing, adaptation, distribution and reproduction in any medium or format, as long as you give appropriate credit to the original author(s) and the source, provide a link to the Creative Commons license, and indicate if changes were made. The images or other third party material in this article are included in the article's Creative Commons license, unless indicated otherwise in a credit line to the material. If material is not included in the article's Creative Commons license and your intended use is not permitted by statutory regulation or exceeds the permitted use, you will need to obtain permission directly from the copyright holder. To view a copy of this license, visit http://creativecommons.org/ licenses/by/4.0/.

(C) The Author(s) 2020 